\title{
THE
}

2017

\section{Heterogeneity in loss aversion: evidence from field elicitations}

Thomas W. Sproul

University of Rhode Island, sproul@uri.edu

Clayton P. Michaud

University of Rhode Island

Follow this and additional works at: https://digitalcommons.uri.edu/enre_facpubs

The University of Rhode Island Faculty have made this article openly available.

Please let us know how Open Access to this research benefits you.

This is a pre-publication author manuscript of the final, published article.

Terms of Use

This article is made available under the terms and conditions applicable towards Open Access Policy Articles, as set forth in our Terms of Use.

\section{Citation/Publisher Attribution}

Sproul, T. and Michaud, C. (2017), "Heterogeneity in loss aversion: evidence from field elicitations", Agricultural Finance Review, Vol. 77 No. 1, pp. 196-216. https://doi.org/10.1108/AFR-05-2016-0045

Available at: https://doi.org/10.1108/AFR-05-2016-0045

This Article is brought to you for free and open access by the Environmental and Natural Resource Economics at DigitalCommons@URI. It has been accepted for inclusion in Environmental and Natural Resource Economics Faculty Publications by an authorized administrator of DigitalCommons@URI. For more information, please contact digitalcommons-group@uri.edu. 


\section{Heterogeneity in loss aversion: evidence from field elicitations}

\section{Structured Abstract}

Purpose: Prospect theory is now widely accepted as the dominant model of choice under risk, but has not been fully incorporated into applied research because of uncertainty about how to include population-level parameter estimates. We aim to characterize heterogeneity across people to lay a foundation for future applied research.

Methodology: We use elicitation data from field experiments in Vietnam to fit a finite Gaussian mixture model using the Expectation Maximization algorithm. Applied results are simulated for investment allocations under myopic loss aversion.

Findings: We find that about $20 \%$ of our sample is classified as extremely loss averse, while the rest of the population is only mildly loss averse. This implies a bimodal distribution of loss aversion in the population.

Research limitations: The data set is only moderately sized: 181 subjects. Future research will be needed to extend these results out of sample, and to other regions.

Value: This paper provides empirical evidence that heterogeneity matters in prospect theory modeling. It highlights how policy makers might be misled by assuming that average prospect theory parameters are typical within the population.

Keywords: behavioral economics, expectation maximization, Gaussian mixture model, heterogeneity, loss aversion, prospect theory

JEL Codes: C38, G02 


\section{Introduction}

Loss aversion is the widespread behavioral phenomenon in which individuals feel losses more strongly than equal-valued gains. It is a key piece of Kahneman and Tversky's (1979) Nobel-prize winning Prospect Theory, and it explains apparent small-scale risk aversion that is otherwise impossible to reconcile with expected utility models (Rabin, 2000). Despite widespread acknowledgement of the importance of loss aversion in behavioral modeling of risky choice, precious little is known about how loss aversion varies across individuals at the population level. Common practice in studies estimating loss aversion (or prospect theory parameters, generally) is to report mean values and standard errors, and possibly the standard deviation or interquartile range, but not to focus on the large amount of variability across people (cf. Kahneman and Tversky, 1979; Tversky and Kahneman, 1991; Abdellaoui, Bleichrodt, and L'Haridon, 2008; Tanaka, Camerer, and Nguyen, 2010; Liu, 2013).

In this article, we estimate a finite Gaussian mixture model to characterize population-level variation in estimated prospect theory parameters, using data from the field experiments of Tanaka, Camerer and Nguyen (2010) with villagers in Vietnam. We find strong evidence in favor of a parsimonious, 2-component mixture model. Our key finding is that approximately $20 \%$ of subjects are classified as extremely loss averse, whereas the remaining majority of the population has milder loss aversion than commonly reported estimates would suggest. In fact, the mixture model allows us to clearly demonstrate that the population-average loss aversion is in no way typical: it is 1.29 standard deviations above the mean for the majority group, and 5.32 standard deviations below the mean for the extremely loss averse group. 
This finding casts serious doubt on the usability of existing parameter estimates for applied modeling. We demonstrate this fact in a numerical exercise highlighting the effect of loss aversion on investment decisions, calibrated to the myopic loss aversion model of Thaler, Kahenman, Tversky, and Schwartz (1997). Our simulations highlight how failing to model loss aversion heterogeneity correctly can lead to mistaken policy conclusions, because the counterfactual of a single-component mixture model implies that far too large a share of the population is moderately loss averse. In particular, we show that policy interventions aimed at reversing the detrimental effects of loss aversion on investors will be highly effective at low levels of intervention, but that the extremely loss averse group will resist treatment at higher levels than expected. These findings suggest that incorporating loss aversion heterogeneity will be a critical piece of future applied prospect theory models.

\section{Background}

Expected utility models traditionally do a poor job predicting true human behavior in many settings, particularly finance and risk management. As a result, there has been a growing push in economic and financial modeling to incorporate advances from the field of behavioral economics, closely accompanied by a large and growing body of evidence demonstrating the improvements obtained by incorporating such elements into consumerchoice models (Chetty, 2015; Madrian, 2014). One of the most prominent contributions of the behavioral literature has been the development of cumulative prospect theory (CPT). These advances include the incorporation of reference-dependent risk preferences, loss aversion and rank- and sign-dependent probability weights into a model of risky 
choice. Despite its recognition as generally improving upon the expected utility model, utilization of the cumulative prospect theory model has only recently found its way into the agricultural economics literature. For example, Babcock (2015) and others (Du et al., 2014; Bougherara and Piet, 2014; Bocquého et al., 2014) have used cumulative prospect theory to explain anomalous crop insurance coverage choices and provide recommendations for improved insurance and policy design.

To fully capture the potential benefits of incorporating prospect theory into financial/risk modeling, however, researchers must have a strong understanding of how the relevant behavioral parameters are distributed at the population level. Naïvely assuming that these parameters are normally and independently distributed can prevent a model from accurately capturing the true distribution of economic behavior. Despite the potential harms of such traditional, simplifying distributional assumptions, there has been little attention in the literature paid to better understanding the underlying distribution of behavioral parameters. One notable contribution in this area is Bruhin, Fehr-Duda, and Epper (2010) who report evidence of heterogeneity among probability weighting parameters among a sample of 448 subjects in Zurich and Beijing collected between 2003-2006 They cleanly (unambiguously) classify subjects into one of three distinct groups: a minority group (roughly 20\%) made up of individuals with risk attitudes consistent with expected utility, and two subgroups of CPT-consistent individuals (roughly $30 \%$ and $50 \%$ ) among which the majority group displays a more moderately Sshaped probability weighting curve relative to the other CPT group.

One critical shortcoming of Bruhin, Fehr-Duda, and Epper's results lie in their elicitation method's inability to measure a coefficient for loss aversion due to the fact that 
the menus used in their experiment contained no mixed lotteries (including both gains and losses) and is therefore not one of the behavioral parameters included in their mixture model. Loss aversion, however, is a crucial component of the prospect theory model that accounts for the fact that losses are often felt with more salience than equivalent gains (in other words, avoiding losses is preferred to acquiring similar gains). Loss aversion can help explain much behavior deemed "irrational" by the expected utility model such as the equity premium puzzle (Benartzi and Thaler, 1995). One key implication of loss aversion is that consumer choices often depend on whether outcomes are framed as gains or losses. Babcock (2015) shows how the finding that farmers prefer lower crop insurance coverage levels despite higher expected net-indemnities at higher coverage levels could be explained by farmers framing insurance decisions/outcomes as one-off gambles (subject to loss aversion) rather than as part of an integrated risk management strategy.

In addition, there is growing evidence of the benefits of incorporating the principle of loss aversion into principal-agent modeling. Fryer, Levitt, List, and Sadoff (2012) present evidence that framing merit pay for teachers in terms of losses can improve student performance. This effect was shown to be equivalent to increasing teacher quality by more than one standard deviation. Engström et al. (2013) find evidence from Sweden that taxpayers are more assiduous about claiming deductions when they owe additional taxes (at the time of filing) than when they expect a refund, suggesting that a tax policy of over-withholding followed by rebates may increase tax revenue. This evidence is consistent with research on the effect of rebates on consumer's purchasing decisions (cf., Read, 2004; Soman, 1998; Loewenstein and Prelec, 1992). 
A richer understanding of how loss aversion is distributed at the population level is also important because of its interaction with the framing of dynamic risk. Loss averse investors who narrowly frame risky decisions/outcomes tend to make shortsighted choices instead of adopting long-term strategies. In addition, such investors will often ignore worthwhile (positive expectation) but risky (non-zero chance of losses) financial opportunities, (Thaler et al., 1997; Kahneman, Wakker, and Sarin, 1997) much to their own detriment. Such behavior is explained in more detail in the numerical application section of this article, following presentation of our estimation results.

To better understand the heterogeneous impacts of loss aversion on decisionmaking at the population level, we use the Expectation Maximization algorithm to estimate a finite Gaussian mixture model of the distribution of CPT parameters. Our data set is sourced from field experiments done by Tanaka, Camerer, and Nguyen (2010), involving 181 rural villagers in Vietnam, in July-August, 2005. We find evidence in support of a 2-component mixture model in which a majority of roughly $80 \%$ of subjects exhibit mild aversion to both risk and loss $(\sigma=0.37, \lambda=1.50)$, while the remaining minority of roughly $20 \%$ of subjects are classified as mildly more risk averse $(\sigma=0.55)$, but extremely loss averse $(\lambda=9.75)$. As a result, estimates of average loss aversion are pulled up dramatically by a small minority of highly loss averse individuals, a finding at odds with the standard convention of reporting mean values of population parameters, as in Tanaka et al. (2010) and Liu (2013), among many others.

We then use this mixture distribution to estimate allocation choices between a high-risk and low-risk investment in the style of the classic Thaler, Tversky, Kahneman, and Schwartz (1997) myopic loss aversion experiment and compare our results to those 
obtained using a single-component (degenerate) mixture model. We conclude with a discussion of implications for policy and potential future research.

\section{A prospect theory mixture model}

Prospect theory is a normative model of consumer preference originally introduced by Kahneman and Tversky in 1979 and subsequently advanced in 1992 under the name cumulative prospect theory (CPT). It is comprised of two independent models, namely a reference-dependent value function, coupled with a probability weighting function. It was designed to account for the observation that i) individuals often display risk-averse behavior when deciding amongst perceived gains while often seeking risk when potential outcomes are perceived as losses, that ii) individuals are more affected by losses than gains (e.g. the disutility generated by a loss of five dollars outweighs the utility derived from a gain of five dollars), and that iii) many individuals systematically misperceive probabilities, underweighting large probabilities while overweighting small ones.

Prospect theory replaces the standard utility function with a reference-dependent utility function (called the value function) that allows for independent risk preferences (curvature of the utility function) for perceived gains and losses; outcomes to the right of an individual's reference point are perceived as gains while outcomes to the left of the reference point are treated as losses. This allows individuals to be risk-averse over gains while simultaneously being risk-seeking over perceived losses, which is generally modeled by a value function that is concave above the reference point, and convex below. The value function also includes a loss aversion parameter, usually denoted $\lambda$, which creates a kink at the reference point and shifts the slope of the value function in the loss 
domain to allow for the commonly observed possibility that the individual is overly affected by losses relative to gains. Loss aversion allows even a risk neutral individual (over both gains and losses) to be unwilling to participate in a positive expectation gamble if the disutility felt from the loss is greater than the utility from the gain. Figure 1 depicts an example of the CPT value function.

$<$ Figure 1 about here $>$

A crucial component of $\mathrm{CPT}$ is the probability weighting function, designed to model the finding that individuals often overweight small probabilities while underweighting large probabilities. Namely, behavioral responses to changes from certainty to near-certainty are too large, and responses to changes in more balanced probabilities (in the range of approximately $0.25-0.75$ ) are too small, to be explained by the value function alone when probabilities are weighted linearly as with expected utility. Figure 2 shows an example of the CPT probability weighting function.

$<$ Figure 2 about here $>$

The net effect of these model components is to induce what is known as the fourfold pattern of risk attitudes, under which individuals are risk-seeking for smallprobability gains and large-probability losses, while being risk-averse for smallprobability losses and large-probability gains (Tversky and Kahneman, 1992). This pattern arises because the overweighting of small probabilities increases the attractiveness of low-probability gains and decreases the attractiveness of low-probability losses, while the underweighting of large probabilities makes higher-probability gains less attractive and higher-probability losses more attractive.

We now develop a mixture model to characterize variation in risk attitudes. As 
mentioned above, our model is designed to be applied to the data set of Tanaka et al. (2010, herein 'TCN'), and we therefore adopt their conventions for the underlying behavioral model. Consider a number of distinct types of people, indexed $c=1, \ldots, C$. Each type makes decisions under risk according to cumulative prospect theory, with typespecific parameters $\lambda_{c}, \alpha_{c}$ and $\sigma_{c} . \lambda_{c}$ is the loss aversion parameter which is identified by a kink in the value function, at the reference point separating gains and losses. $\alpha_{c}$ is the parameter describing overweighting of low probabilities in Prelec's (1998) axiomatically derived, compound invariant probability weighting function, $w_{c}(p)=\exp \left(-(\ln 1 / p)^{\alpha_{c}}\right) \cdot \sigma_{c}$ is the curvature parameter for the value function, which we assume to be consistent across both gains and losses for consistency with TCN. We modify the TCN formulation slightly, in the fashion of Liu (2013), to make $\sigma_{c}$ comparable to the coefficient of relative risk aversion (CRRA) from the familiar powerlaw and logarithmic expected utility functions. Specifically, we define the class-specific value function according to:

$$
v_{c}(x)= \begin{cases}(x-r)^{1-\sigma_{c}} & \text { if } x \geq r \\ -\lambda_{c}(r-x)^{1-\sigma_{c}} & \text { if } x<r\end{cases}
$$

where $r$ is the prospect theory reference point, which is commonly normalized to zero in experimental elicitation procedures. For cases where $\sigma_{c}=1$, we use $\ln x$ for the value function according to common practice for CRRA utility.

Thus, for each class $c$, if we consider a prospect with two outcomes, the prospect is valued according to

$$
V=w\left(p_{1}\right) v\left(x_{1}\right)+\left(1-w\left(p_{1}\right)\right) v\left(x_{2}\right)
$$


for $x_{1} x_{2}>0$, where $\left|x_{1}\right|>\left|x_{2}\right|$ is assumed without loss, or $V=w\left(p_{1}\right) v\left(x_{1}\right)+w\left(p_{2}\right) x_{2}$ otherwise (that is, in the case of a 'mixed gamble,' when one outcome is a loss). Using this specification, TCN outline a new methodology to experimentally estimate (or elicit) parameters within individuals, according to the model described above. We use this methodology to transform a data set comprised of observations of experimental choices into a data set of observations of parameters for each individual subject, denoted $\lambda_{i}, \alpha_{i}$ and $\sigma_{i}$.

We use the individual parameter estimates to fit a mixture model, with the goal of classifying individuals into distinct types, each of which is expressed as a multivariate normal distribution of the $\lambda, \alpha$ and $\sigma$ parameters, with associated mean vector and covariance matrix. Thus, our specification is general enough to identify heterogeneity of risk attitudes on average across types, as well as the degree to which each type might include relatively broad or narrow ranges of parameter values. As will be seen below, we identify dramatic differences in loss aversion across types, but do not find as much contrast in the marginal distributions of the other parameters.

The mixture model is fit using the well-known expectation maximization (EM) algorithm of Dempster, Laird and Rubin (1977). EM is a Bayesian procedure that uses the likelihood function and the mixture probabilities, $\pi_{c}$, to update so-called 'membership probabilities,' $\tau_{i c}$, for each individual $i$, for each type (or 'class') $c$. The average of these membership probabilities becomes the updated mixture probability for each class, $\pi_{c}$, which completes the E-step (expectation) of the algorithm. In the M-step (maximization), the updated mixture probabilities are held fixed while the log likelihood 
is maximized by varying the parameters for each class, collectively referred to as the vector, $\theta_{c}$. After the M-step, the algorithm repeats until suitable convergence is achieved.

Formally, let $t$ denote the most recent iteration of the algorithm. Then the updated mixture probabilities for step $t+1$ are given by

$$
\tau_{i c}^{t+1}=\frac{\pi_{c}^{t} \cdot f\left(x_{i} \mid \hat{\theta}_{c}^{t}\right)}{\sum_{j=1}^{C} \pi_{j}^{t} \cdot f\left(x_{i} \mid \hat{\theta}_{j}^{t}\right)},
$$

for each class. Here, $x_{i}$ are the data for individual $i, \hat{\theta}_{c}^{t}$ are the most recent maximum likelihood estimates for the parameters describing type $c$, and $f$ denotes the probability of the data given the estimated parameters. The updated mixture probabilities for each type (at the population level) are then simply the averages of the membership probabilities:

$$
\pi_{c}^{t+1}=\frac{1}{I} \sum_{i=1}^{I} \tau_{i c}^{t+1} .
$$

The maximization step then maximizes the log-likelihood function, taking the mixture probabilities as fixed. The updated estimate, $\hat{\theta}^{t+1}$, solves

$$
\max _{\theta} L L(\theta ; \pi)=\sum_{i=1}^{I} \ln \left(\sum_{c=1}^{C} \pi_{c}^{t+1} \cdot f\left(x_{i} \mid \theta_{c}\right)\right) .
$$

Recall that $\theta_{c}$ represents the parameters collectively describing the multivariate normal for type $c$. Since our behavioral model is a 3-parameter model, each $\theta_{c}$ contains three mean parameters, as well as three additional parameters to populate the covariance matrix, which we assume to be diagonal following Bruhin, Fehr-Duda and Epper (2010). The characteristics of the mixture components are not the only useful information arising from the EM procedure. The final membership probabilities, $\tau_{i c}$, each represent the posterior probability that individual $i$ belongs to class $c$, a measurement which can be used not only in maximizing the likelihood function but also in evaluating the 
performance of the mixture model as a classifier. This information is particularly useful in cases where the number of component distributions (mixture components) is not empirically known ex ante, because it can then be used for model selection.

The next section discusses the data set used in our Estimation section, followed by a section covering model selection and evaluation of results.

\section{Data}

As the desire to incorporate elements of cumulative prospect theory in to economic modeling has increased, so has the desire for accurate CPT parameter estimates at the individual level. Obtaining such estimates poses a unique challenge to researchers for a number of reasons. One such challenge arises from the fact that CPT parameters must be (at least to some extent) estimated simultaneously since certainty equivalents/switching points are a function of both risk preferences and probability weighting in the case of non-mixed gambles, as well as by the loss aversion parameter for the case of mixed gambles. A method that has been gaining popularity in recent years is presented in Tanaka, Camerer, and Nguyen (2010). The TCN method uses switching-points from three pairwise lottery choice menus in the style of Holt and Laury $(2002,2005)$ to exactly identify estimates of the CPT parameters within individuals.

To estimate the three CPT parameters, TCN designed three series of paired lotteries as shown in Table 1. Each row is a choice between two binary lotteries with probabilities expressed using bingo balls. To ensure monotonic choices, subjects are asked at which question they would like to "switch" from Option A to option B in each series. Once the three series (over a total of 35 choices) are complete, one of 35 
numbered balls was drawn from a bingo cage and the selected lottery was played using 10 numbered balls.

$<$ Table 1 about here $>$

The menus are designed in such a way that any combination of the three choices determines a particular interval of CPT parameter values, illustrated in Table 2. The switching point in Series 1 and 2 are used to jointly estimate $\sigma$ and $\alpha$. $\lambda$ is determined by the switching point in Series 3 based on $\sigma$ calculated from Series 1 and 2. Series 3 was designed to take similar values of $\lambda$ across different levels of $\sigma$, while $\alpha$ is differenced out of the Series 3 calculation by setting all outcomes as equally probable.

$<$ Table 2 about here $>$

TCN conducted such an experiment in July-August 2005 with 181 villagers in rural Vietnam. Vietnam was chosen as the field site because most Vietnamese villagers are poor but literate, so they can be easily motivated with modest financial stakes while also comprehending experimental instructions. Their experiment lasted roughly four hours (including survey questions and other exercises; the risk elicitation takes 20 minutes or less) and average total earnings were 174,141 dong (about 11 dollars), or roughly 6 to 9 days' earnings for casual unskilled labor. The data used in our analysis is from the reported choices in the publicly available replication data provided by TCN. Details about the distributions of parameter estimates are provided below.

\section{Estimation and results}

To allow for, and identify, heterogeneity of preferences across individuals, we estimate a finite Gaussian mixture model on the data set of estimated prospect theory parameters 
using the Expectation Maximization (EM) algorithm of Dempster, Laird and Rubin (1977). A known shortcoming of the EM algorithm is that the researcher must specify the number of mixture types. While the algorithm has some capacity to 'zero out' redundancies as it endogenously determines the number and nature of types, in practice, specification of too many mixture components can lead to over-fitting. Information criteria such as the corrected Akaike information criterion (AICc) or the Bayesian information criterion (BIC), or comparable cross-validation approaches, are often used for model selection but may be inadequate for choosing between mixture models due to insufficient penalization of extra model parameters (Celeux and Soromenho, 1996; Biernacki, Celeux and Govaert, 2000). The latter authors introduce the integrated completed likelihood criterion (ICL), a modification of BIC with an additional penalty for mean entropy. More entropy means more ambiguous assignments to the various classes, corresponding to $\tau_{i c}$ values that are not close to either zero or one, an indication that the fitted mixture model is not serving as an effective classifier of the data into distinct types. Thus, ICL adjusts BIC for the quality of classifications, and this quality is conveniently measured by posterior membership probabilities estimated in the course of the EM procedure. Table 3 shows estimates for AICc, BIC and ICL, for models fitted with up to four mixture components.

$<$ Table 3 about here $>$

Table 3 shows two things: first, all three selection criteria indicate that a mixture of at least two components is preferred to a model of only one component distribution. This helps justify the mixture model approach. Second, both BIC and ICL indicate that adding components beyond two does not result in enough additional information to 
justify the inclusion of the extra parameters in the model (note that the values are only comparable within columns, not across them, and that lower is better). However, the AICc results do not agree with the other two criteria. While criticisms of AICc's consistency (vs. BIC) exist as mentioned above, we cannot rule out AICc out of hand because the literature remains divided as to whether or when BIC outperforms AIC/AICc, especially in cases where BIC is likely to under-fit models in finite samples (Burnham and Anderson, 2004).

To address this issue, we plot the distributions of posterior membership probabilities for the 2-component model and the 4-component model in Figure 3 and Figure 4, respectively. We also show classification statistics in Table 4 for each of the 2component and 4-component fitted mixture models. The $\tau_{i c} \geq 0.99$ column gives the number of observations (and percent of the total) that exhibit $\tau_{i c} \geq 0.99$ for at least one class, $c$, indicating a posterior probability of membership in a single class of at least $99 \%$. The $95 \%$ and $90 \%$ columns are defined similarly. It is apparent that the 2-component model dramatically outperforms the 4-component model in terms of assigning observations to genuinely distinct types. For this reason, along with a preference for a more parsimonious model barring strong evidence to the contrary, we consider the 2component model estimates as our primary results. Still, we contrast it against the 1component (degenerate mixture) model and the 4-component model in the numerical application following this section. The 2-component model captures critical type information that a single component distribution cannot distinguish, while at the same time providing near identical information to the higher resolution 4-component model in terms of the implied distribution over risky choices in the population. 
$<$ Figure 3 about here $>$

$<$ Figure 4 about here $>$

$<$ Table 4 about here $>$

Figures 3 and 4, and Table 4 all show that the more parsimonious 2-component mixture model is also a more effective classifier on our data set. We can see that the 2component mixture encodes substantially more population level information than a single-component model, but the 4-mixture does not add much information beyond what is captured with two components. Figure 5 demonstrates this fact by plotting the marginal kernel densities across each of the prospect theory parameters for each fitted model.

$<$ Figure 5 about here $>$

Figure 5 also demonstrates our key finding, enabled by the mixture modeling approach: the right-hand panel (for the loss aversion parameter, $\lambda$ ) shows that the average level of loss aversion is not as informative as population level statistics for the other prospect theory parameters because it is not a typical level of loss aversion. Our estimated model shows that almost $20 \%$ of the population are extremely loss averse (Group I), with estimated parameters centered around $\lambda=9.75$, while over $80 \%$ of the population (Group II) have loss aversion levels in a range centered on $\lambda=1.50$, lower than the classical value of $\lambda=2.25$ reported by Kahneman and Tversky (1979), and used ubiquitously ever since. The population-level marginal distribution arising from the mixture is clearly bimodal, and the population-average value of $\lambda=2.91$ is 1.29 standard deviations above the mean for the majority group (Group II), yet it is 5.32 standard deviations below the mean for the extremely loss averse minority group (Group I).

$<$ Table 5 about here $>$ 
Table 5 shows our estimation results for the 2-component mixture model. The key, separating feature between the two groups is loss aversion, with little overlap between groups indicating a clean separation by virtue of the EM algorithm acting as an effective classifier. We note that the extremely loss averse group is on average more risk averse (by this we mean a higher curvature parameter, $\sigma$ ), but the distributions of this parameter have near-identical support across the two groups in our sample. All estimated model parameters were statistically significantly different between Group I and Group II at the 99.9\% level, except for the standard deviation parameter for the marginal distribution of the probability weighting parameter, $\alpha(p=0.089)$.

We also note that the two groups appear to be nearly identical with respect to the probability weighting parameter, $\alpha$, a finding that at first glace appears to stand in contrast to the results of Bruhin et al. (2010), who identify groups primarily based on differences in probability weighting behavior. Both their experimental methods and behavioral model differ from ours, however, in that their data set contains many more observations per subject, allowing for a richer model of probability weighting that includes the two-parameter weighting function of Goldstein and Einhorn (1987) which differentiates between the function's "curvature" and "elevation" and has been shown to account well for individual heterogeneity (Wu, Zhang, and Gonzalez, 2004). Still, this contrast probably merits further research. Figure 6 shows the fitted 2-component mixture model overlaid on a histogram of parameter estimates for each of the prospect theory parameters. The bimodal distribution of loss aversion parameters is clearly visible.

$<$ Figure 6 about here $>$ 
We thank a referee for pointing out that intuitively our results regarding extreme loss aversion classify a percentage of the population as having an "essentially inverse Lshaped" value function, which might be considered a form of extreme risk aversion. To check whether this intuition would hold in an expected utility setting, we re-ran our analysis with un-weighted probabilities and a constant relative risk aversion utility function with parameter $r$ fitted by maximum likelihood. First, we found no "separation" in the parameter values when applying our goodness of fit criteria for mixture models as above: the ICL criterion dramatically preferred no mixture to any mixture with $C>1$ components. Second, we found that the maximum likelihood estimate of $r$ under expected utility was a better predictor (via OLS) of the sigma curvature parameter (adjusted $\mathrm{R}^{2}=$ 0.613 ) than of the lambda loss aversion parameter (adjusted $\mathrm{R}^{2}=0.311$ ). Both regressions had constant terms and coefficient estimates significant at the $99.9 \%$ level, but the regression for sigma had t-statistics approximately 1.5 times larger in magnitude. Finally, we found that the TCN lambda parameter was uncorrelated with the root mean squared error of the fitted estimate $r$, so noise in the data does not explain the lack of replication in an expected utility setting. We conclude that the intuitive observation of an inverse L-shaped value function does not translate directly to a mixture with an extreme risk aversion group under expected utility, likely because of probability weights being an essential component of the CPT model.

\section{Application: myopic loss aversion and investment}

Myopic loss aversion (MLA) is the behavioral phenomenon used to explain the equity premium puzzle, and it is responsible for misallocation of retirement and investment 
portfolios worldwide (Benartzi and Thaler, 1995). Its name refers to the combined effect/result of loss aversion and narrow framing. Narrow framing is a key concept from the mental accounting literature (Thaler, 1999) that refers to how decisions and outcomes are aggregated (framed) both cross-sectionally (across simultaneous outcomes) and intertemporally (across consecutive outcomes). Loss averse investors who narrowly frame risky decisions/outcomes tend to make shortsighted choices instead of adopting long-term strategies. In addition, as stated earlier, such investors will often ignore worthwhile (positive expectation) but risky (non-zero chance of losses) financial opportunities, much to their own detriment. Narrow framing of decisions and narrow framing of outcomes tend generally tend to go together, and it is the combination of both tendencies that defines a myopic investor (Thaler et al., 1997).

Put another way, the myopia component of MLA leads to improperly aggregating a sequence of investments or positive-expectation bets over time, leading to inflated perceptions of the potential for, and probability of, losses occurring. These perceptions interact with loss aversion, in which losses are felt more strongly than equal-sized gains, to cause investors to avoid opportunities that they would take over longer time horizons. The MLA phenomenon has been extensively verified in experimental settings, and has even been found in professional futures traders (Haigh and List, 2005).

The behavioral literature has also identified cross-sectional and inter-temporal forms of myopia. In the case where two coin flips are carried out simultaneously, a failure to aggregate over the two independent outcomes is an example of cross-sectional myopia. Another example of cross-sectional myopia is found in the experimental results of Benjamin, Rabin and Raymond (2016) who model "non-belief in the law of large 
numbers," a phenomenon in which individuals fail to account for the changing statistical properties of aggregated, independent random variables. For example, individuals might use something like a mistaken proportionality heuristic when they assume that the probability of seven or more heads in ten coin flips (17.2\%) is about equal to the probability of 70 or more heads in 100 coin flips ( $0.003 \%)$. In the case of consecutive coin flips, such a failure would be an example of inter-temporal myopia, which is directly related to the frequency with which decisions or outcomes are evaluated. Since the probability of observing a loss is higher when outcomes (e.g. stock returns) are evaluated more frequently, the utility experienced from owning stocks will be lower for more myopic loss averse investors (Kahneman, Wakker, and Sarin, 1997) who should be expected over time to gravitate towards lower levels of risk (Thaler, et al., 1997).

To demonstrate the importance of the heterogeneity we have identified in the loss aversion parameter, we conduct a numerical exercise based on the experimental setting used to evaluate myopic loss aversion (MLA) in Thaler et al. (1997). Their experiment is essentially a fund management exercise where subjects need to allocate their portfolios between a simulated equity fund designed to resemble the returns profile of the S\&P 500, and a simulated bond fund designed to resemble the returns profile of 5-year treasuries. If subjects are myopic and loss averse, then the setup predicts that the feedback frequency will affect their allocation between the two funds: they are not told which is which, and more frequent monitoring will expose them to relatively more losses in stocks, triggering more loss averse subjects to allocate more heavily towards bonds.

Fundamentally, the myopic loss aversion result is about the effect of loss aversion on a distribution of investment returns over time - due to myopia, the subjects do not 
realize the statistical properties of aggregating positive-expectation gambles, and so do not realize that they can commit for longer periods than the assigned experimental feedback frequency. Thaler et al. assign subjects to be able to adjust their portfolios as frequently as every month, or as infrequently as every five years. Theory suggests a fiveyear adjustment frequency is likely to be sufficiently long to inhibit the effects of loss aversion (by lowering the probability of a loss in the equities fund), an estimate that we confirm in our numerical exercise below.

However, a key distinction we find is that the majority of the population (Group II) is far less affected by loss aversion, and are induced to optimal long-term investment (100\% allocated to stocks, in this example) by adjustment frequencies of one year or less. The contrast of optimal one-year adjustments vs. five-year adjustments is a striking result made possible by our estimation of heterogeneity in a mixture model. In their original work identifying myopic loss aversion as a possible cause of the equity premium puzzle, Benartzi and Thaler (1995) hypothesize that while theory predicts five-year adjustment periods to be optimal for investment planning in the face of MLA, the equity premium puzzle is likely observed in part because investors need to evaluate their portfolios at least once per year at tax time, and many evaluate at least quarterly due to the common practice of quarterly statements being issued. Our results suggest that a disproportionate share of the MLA effect of under-allocation of investments to stocks is due to a relatively small share of the population operating under extreme loss aversion.

To apply our results in an MLA setting, we use the experimental setup of Thaler et al. who calibrate quarterly log returns of the S\&P 500 to be $2 \%$ with a $5 \%$ standard deviation (these are intended to be real returns), and quarterly log returns of the bond 
fund to be $0.5 \%$ with a $0.25 \%$ standard deviation. To enable comparison with their results we apply a linear loss aversion model in which probability weights and value function curvature are ignored. Figure 7 shows the effect of linear loss aversion on the implied distribution of stock returns, using a loss aversion coefficient of $\lambda=2.25$ and Thaler et al.'s (1997) calibrated returns distribution, at different feedback frequencies. The Figure clearly shows how loss averse investors would experience dramatically more losses in shorter time frames, and so might shy away from allocating investments to the stock market if they weight losses more heavily than gains of equal size.

$<$ Figure 7 about here $>$

Table 6 shows summary statistics for the simulated distribution of cumulative 30year investment returns under MLA, broken down by group (from our estimated 2component mixture model) and by feedback frequency. The majority group (Group I) with standard prospect theory parameters achieves dramatically higher returns at nearly every feedback frequency, and notably, achieves maximum returns at the one-year frequency. The extreme loss aversion group (Group II), on the other hand, dramatically over-allocates to the bond fund and does not even double their money on average over a 30-year horizon if feedback is given at a quarterly or higher frequency. For any (perhaps, "libertarian paternalistic") interventions that might be considered, the extreme loss aversion group should clearly be targeted, as they appear to bear the bulk of the harm that comes from MLA-influenced investment.

$$
<\text { Table } 6 \text { about here }>
$$

We can also use this numerical exercise to show how researchers ignoring the possibility of a mixture model might be misled by using population-average parameter 
measures for inference, or to guide policy. In particular, we compare the degenerate, 1component mixture model estimated above against results from the 2-component model presented here. Because the 1-component model cannot account for the bimodal nature of loss aversion in the population, the average, typical individual in the 1-component model is definitely not typical under the 2-component mixture. The effect of this mischaracterization is to overstate the effects of MLA with more frequent feedback but to understate its importance when feedback is infrequent. This can be seen in Figure 8, which shows the allocation percentage to stocks over a simulated population from each model. The single-component model overstates the effect of MLA for feedback frequencies from weekly up to semi-annually, but then understates the effect of MLA for longer feedback frequencies.

$<$ Figure 8 about here $>$

Effectively, then, an applied researcher using a single-component model would predict that loss aversion is more important of a decision factor to the bulk of the population than it actually is. The majority group exhibits only mild loss aversion, experiences relatively mild effects on investment outcomes, and converges to optimal behavior over shorter time frames than would be predicted using the population average loss aversion estimate. On the other hand, Group I (extremely loss averse) experiences severe effects on their investment outcomes and are much more resistant to lengthening the feedback window. It is not depicted in Figure 8, but Group I's allocations converge sharply towards optimal allocations between the one- and two-year feedback intervals.

\section{Discussion}


In this article, we have shown how modeling heterogeneity is a critical piece of applied behavioral modeling. Namely, while we find little variation across prospect theory 'types' in terms of risk aversion (curvature of the value function) and probability weighting, we find dramatic variation across types in terms of the level of estimated loss aversion. Approximately twenty percent of our sample is classified as extremely loss averse in a parsimonious, 2-component finite Gaussian mixture model. While about eighty percent of the population is classified as mildly loss averse, researchers ignoring this heterogeneity structure would instead assume that moderate loss aversion is typical, because of how much the extremely loss averse group drags up the average parameter estimate. Our results show that moderate loss aversion, while average in terms of estimated loss aversion parameters, actually applies to only ten or twenty percent of the population depending on how it is defined.

We extend these findings in a numerical simulation exercise using the myopic loss aversion investment model of Thaler et al. (1997). The effect of myopia, when combined with loss aversion, is to cause investors to avoid worthwhile risky investments in favor of safer ones with almost no real returns, especially when they consider their investment decisions over shorter and shorter time frames. When we use the fitted mixture model in the simulation, we find that longer investment feedback frequencies mitigate the effects of loss aversion, but in a manner slightly different than expected. Namely, for the majority group of individuals classified as mildly loss averse, we find that lengthening investment feedback intervals to only one year corrects the myopic loss aversion investment problem entirely, and their investment losses for shorter feedback intervals are relatively minor relative to those of the extremely loss averse group. For the 
minority group, lengthening feedback intervals has relatively little effect on their investment allocations up to one year, but they then experience a discontinuous jump where their allocations converge to those of the majority group for intervals of two years or longer.

Overall, our results highlight the importance of heterogeneity in applied modeling, and of considering the possibility of discrete behavioral types within the population. A promising area of future applied research in agricultural finance and risk modeling is to extend of our results to the crop insurance domain. In a recent article, Babcock (2015) used a prospect theory model to show evidence that U.S. farmers' crop insurance adoptions likely arise from myopic treatment of crop insurance payouts as a standalone investment. If farmers come from distinct types with respect to loss aversion, then this will figure dramatically into their choices of coverage levels and acreage enrolled in the program. Since crop insurance subsidies are the primary mechanism for transferring income to the U.S. farm sector, the heterogeneity we have identified implies there will be winners and losers among American farmers, driven by their preferences for risk and loss. With crop insurance premiums reaching \$11.1 billion in 2012 and with average premium subsidies above $60 \%$, whether or how crop insurance rates might be structured to mitigate effects on extremely loss averse farmers is a policy question of national importance.

\section{References}

Abdellaoui, M., Bleichrodt, H., \& l'Haridon, O. (2008). A tractable method to measure utility and loss aversion under prospect theory. Journal of Risk and Uncertainty, 36(3), 245-266. 
Benartzi, S., \& Thaler, S. (1995). Myopic loss aversion and the equity risk premium puzzle. Quarterly Journal of Economics, 110(1).

Benjamin, D. J., Rabin, M., \& Raymond, C. (2016). A model of nonbelief in the law of large numbers. Journal of the European Economic Association, 14(2), 515-544.

Biernacki, C., Celeux, G., \& Govaert, G. (2000). Assessing a mixture model for clustering with the integrated completed likelihood. Pattern Analysis and Machine Intelligence, IEEE Transactions on, 22(7), 719-725.

Bocquého, G., Jacquet, F., \& Reynaud, A. (2014). Expected utility or prospect theory maximisers? Assessing farmers' risk behaviour from field-experiment data. European Review of Agricultural Economics, 41(1), 135-172.

Bougherara, D., \& Piet, L. (2014, August). The impact of farmers' risk preferences on the design of an individual yield crop insurance. In XIVth Congress of the European Association of Agricultural Economists, Ljubljana (Slovenia), August (pp. 26-29).

Bruhin, A., Fehr-Duda, H., \& Epper, T. (2010). Risk and rationality: Uncovering heterogeneity in probability distortion. Econometrica, 78(4), 1375-1412.

Burnham, K. P., \& Anderson, D. R. (2004), "Multimodel inference: understanding AIC and BIC in Model Selection." Sociological Methods \& Research 33 (2), 261-304.

Celeux, G., \& Soromenho, G. (1996), "An Entropy Criterion for Assessing the Number of Clusters in a Mixture Model," Journal of Classification 13, 195-212.

Chetty, R. (2015). Behavioral economics and public policy: A pragmatic perspective (No. w20928). National Bureau of Economic Research. 
Dawes, J. (2004). "Price changes and defection levels in a subscription-type market: can an estimation model really predict defection levels?", Journal of Services Marketing, Vol. 18 Iss: 1, pp.35 - 44

Dempster, A.P., Laird, N.M., \& Rubin, D.B. (1977). "Maximum Likelihood from Incomplete Data via the EM Algorithm." Journal of the Royal Statistical Society, Series B 39 (1), 1-38.

Du, X., Feng, H., \& Hennessy, D. A. (2014). Rationality of Choices in Subsidized Crop Insurance Markets.

Engström, P., Nordblom, K., Ohlsson, H., \& Persson, A. (2015). Tax compliance and loss aversion. American Economic Journal: Economic Policy,7(4), 132-164.

Fryer Jr, R. G., Levitt, S. D., List, J., \& Sadoff, S. (2012). Enhancing the efficacy of teacher incentives through loss aversion: A field experiment (No. w18237). National Bureau of Economic Research.

Goldstein, W. M., \& Einhorn, H. J. (1987). Expression theory and the preference reversal phenomena. Psychological review, 94(2), 236.

Haigh, M. S., \& List, J. A. (2005). Do professional traders exhibit myopic loss aversion? An experimental analysis. The Journal of Finance, 60(1), 523-534.

Holt, C. A., \& Laury, S. K. (2002). Risk aversion and incentive effects.American economic review, 92(5), 1644-1655.

Holt, C. A., \& Laury, S. K. (2005). Risk aversion and incentive effects: New data without order effects. The American economic review, 95(3), 902-904.

Kahneman, D., \& Tversky, A. (1979). Prospect theory: An analysis of decision under risk. Econometrica: Journal of the Econometric Society, 263-291. 
Kahneman, D., Wakker, P. P., \& Sarin, R. (1997). Back to Bentham? Explorations of experienced utility. The quarterly journal of economics, 375-405.

Liu, E. M. (2013). Time to change what to sow: Risk preferences and technology adoption decisions of cotton farmers in China. Review of Economics and Statistics, 95(4), 1386-1403.

Loewenstein, G., \& Prelec, D. (1992). Anomalies in intertemporal choice: Evidence and an interpretation. The Quarterly Journal of Economics, 573-597.

Madrian, B. C. (2014). Applying insights from behavioral economics to policy design (No. w20318). National Bureau of Economic Research.

Prelec, D. (1998). The probability weighting function. Econometrica, 497-527.

Rabin, M. (2000). Risk aversion and expected-utility theory: A calibration theorem. Econometrica, 68(5), 1281-1292.

Read, D. (2004). Intertemporal choice. Blackwell handbook of judgment and decision making, 424-443.

Samuelson, Paul A., "Risk and Uncertainty: A Fallacy of Large Numbers," Scientia, XCVIII (1963), 108-13.

Tanaka, T., Camerer, C. F., \& Nguyen, Q. (2016). Risk and time preferences: linking experimental and household survey data from Vietnam. In Behavioral Economics of Preferences, Choices, and Happiness (pp. 3-25). Springer Japan.

Thaler, R. H. (1999). Mental accounting matters. Journal of Behavioral decision making, 12(3), 183. 
Thaler, R. H., Tversky, A., Kahneman, D., \& Schwartz, A. (1997). The effect of myopia and loss aversion on risk taking: An experimental test. The Quarterly Journal of Economics, 647-661.

Tversky, A., \& Kahneman, D. (1991). Loss aversion in riskless choice: A referencedependent model. The quarterly journal of economics, 1039-1061.

Tversky, A., \& Kahneman, D. (1992). Advances in prospect theory: Cumulative representation of uncertainty. Journal of Risk and uncertainty,5(4), 297-323.

Wu, G., Zhang, J., \& Gonzalez, R. (2004). Decision under risk. Blackwell handbook of judgment and decision making, 399423. 
Tables

Table 1. Three Series of Pairwise Lotteries (in 1,000 dong) from Tanaka et al. (2010)

\begin{tabular}{|c|c|c|c|c|}
\hline \multicolumn{2}{|c|}{ Option A } & \multicolumn{2}{|c|}{ Option B } & \multirow[t]{2}{*}{ Expected payoff difference $(\mathrm{A}-\mathrm{B})$} \\
\hline Series I & & & & \\
\hline Balls 1-? & Balls 4-10 & Ball 1 & Balls 2-10 & \\
\hline 40 & 10 & 68 & 5 & 7.7 \\
\hline 40 & 10 & 75 & 5 & 7.0 \\
\hline 40 & 10 & 83 & 5 & 6.0 \\
\hline 40 & 10 & 93 & 5 & 5.2 \\
\hline 40 & 10 & 106 & 5 & 3.9 \\
\hline 40 & 10 & 125 & 5 & 2.0 \\
\hline 40 & 10 & 150 & 5 & -0.5 \\
\hline 40 & 10 & 185 & 5 & -4.0 \\
\hline 40 & 10 & 220 & 5 & -7.5 \\
\hline 40 & 10 & 300 & 5 & -15.5 \\
\hline 40 & 10 & 400 & 5 & -25.5 \\
\hline 40 & 10 & 600 & 5 & -45.5 \\
\hline 40 & 10 & 1,000 & 5 & -85.5 \\
\hline 40 & 10 & 1,700 & 5 & -155.5 \\
\hline
\end{tabular}

Series 2

Balls 1-9

40

40

40

40

40

40

40

40

40

40

40

40

40

40
Ball 10

30

30

30

30

30

30

30

30

30

30

30

30

30

30

Series 3

Balls 1-5 Balls 6-10

$\begin{array}{cc}25 & -4 \\ 4 & -4 \\ 1 & -4 \\ 1 & -4 \\ 1 & -8 \\ 1 & -8 \\ 1 & -8\end{array}$

\section{Balls 1-7 Balls 8-10}

$\begin{array}{rl}54 & 5 \\ 56 & 5 \\ 58 & 5 \\ 60 & 5 \\ 62 & 5 \\ 65 & 5 \\ 68 & 5 \\ 72 & 5 \\ 77 & 5 \\ 83 & 5 \\ 90 & 5 \\ 100 & 5 \\ 110 & 5 \\ 130 & 5\end{array}$

| -0.3

$-1.7$

$-3.1$

$-4.5$

$-5.9$

$-8.0$

$-10.1$

$-12.9$

$-16.4$

$-20.6$

$-25.5$

$-32.5$

$-39.5$

$-53.5$

Balls 1-5 Balls 6-10

$\begin{array}{ll}30 & -21 \\ 30 & -21 \\ 30 & -21 \\ 30 & -16 \\ 30 & -16 \\ 30 & -14 \\ 30 & -11\end{array}$

6.0

$-4.5$

$-6.0$

$-8.5$

$-10.5$

$-11.5$

$-13.0$ 
Table 2. Switching-point Based Parameter Estimates

Question at which preference switches from Option A to Option B, and approximations of $\alpha, \sigma$ and $\lambda$.

Series I (questions $1-14$ )

\begin{tabular}{|c|c|c|c|c|c|c|c|}
\hline$\sigma^{\alpha}$ & 0.4 & 0.5 & 0.6 & 0.7 & 0.8 & 0.9 & 1.0 \\
\hline 0.2 & 9 & 10 & 11 & 12 & 13 & 14 & never \\
\hline 0.3 & 8 & 9 & 10 & 11 & 12 & 13 & 14 \\
\hline 0.4 & 7 & 8 & 9 & 10 & 11 & 12 & 13 \\
\hline 0.5 & 6 & 7 & 8 & 9 & 10 & 11 & 12 \\
\hline 0.6 & 5 & 6 & 7 & 8 & 9 & 10 & 11 \\
\hline 0.7 & 4 & 5 & 6 & 7 & 8 & 9 & 10 \\
\hline 0.8 & 3 & 4 & 5 & 6 & 7 & 8 & 9 \\
\hline 0.9 & 2 & 3 & 4 & 5 & 6 & 7 & 8 \\
\hline 1.0 & 1 & 2 & 3 & 4 & 5 & 6 & 7 \\
\hline
\end{tabular}

Series 2 (questions $15-28$ )

Series 3 (questions 29-35)

\begin{tabular}{lccc} 
Switching question & $\sigma=0.2$ & $\sigma=0.6$ & $\sigma=1$ \\
\hline 1 & $\lambda>0.14$ & $\lambda>0.20$ & $\lambda>0.29$ \\
2 & $0.14<\lambda<1.26$ & $0.20<\lambda<1.38$ & $0.29<\lambda<1.53$ \\
3 & $1.26<\lambda<1.88$ & $1.38<\lambda<1.71$ & $1.53<\lambda<1.71$ \\
4 & $1.88<\lambda<2.31$ & $1.71<\lambda<2.25$ & $1.71<\lambda<2.42$ \\
5 & $2.31<\lambda<4.32$ & $2.25<\lambda<3.73$ & $2.42<\lambda<3.63$ \\
6 & $4.32<\lambda<5.43$ & $3.73<\lambda<4.82$ & $3.63<\lambda<4.83$ \\
7 & $5.43<\lambda<9.78$ & $4.82<\lambda<9.13$ & $4.83<\lambda<9.67$
\end{tabular}

Note: Bold indicates choices compatible with $\mathrm{EU}(\alpha=1)$ and risk aversion. 
Table 3. Information criteria for model selection

\begin{tabular}{cccc} 
Components & AICc & BIC & ICL \\
\hline 1 & 1100.89 & 1120.01 & 560.01 \\
2 & 872.88 & $\mathbf{9 1 4 . 2 3}$ & $\mathbf{5 4 2 . 8 2}$ \\
3 & 853.43 & 916.92 & 637.31 \\
4 & $\mathbf{8 4 0 . 7 0}$ & 926.23 & 691.56
\end{tabular}


Table 4. Quality of posterior classification

\begin{tabular}{ccrrr} 
Components & Obs. & \multicolumn{1}{c}{$\tau_{i c} \geq 0.99$} & \multicolumn{1}{c}{$\tau_{i c} \geq 0.95$} & \multicolumn{1}{c}{$\tau_{i c} \geq 0.90$} \\
\hline 2 & 181 & $179(98.90 \%)$ & $181(100.0 \%)$ & $181(100.0 \%)$ \\
4 & 181 & $35(19.34 \%)$ & $56(30.94 \%)$ & $78(43.09 \%)$
\end{tabular}


Table 5. Estimation Results for the 2-Component Mixture

\begin{tabular}{|c|c|c|c|c|}
\hline \multirow{3}{*}{ Weight } & \multicolumn{2}{|c|}{ Group I } & \multicolumn{2}{|c|}{ Group II } \\
\hline & \multicolumn{2}{|c|}{$\begin{array}{l}0.1713 \\
(0.0291)\end{array}$} & \multicolumn{2}{|c|}{$\begin{array}{l}0.8287 \\
(0.0291)\end{array}$} \\
\hline & Mean & Std & Mean & Std \\
\hline$\alpha$ & $\begin{array}{l}0.7728 \\
(0.0488)\end{array}$ & $\begin{array}{l}0.2722 \\
(0.0183)\end{array}$ & $\begin{array}{l}0.7400 \\
(0.0208)\end{array}$ & $\begin{array}{l}0.2575 \\
(0.0091)\end{array}$ \\
\hline$\sigma$ & $\begin{array}{l}0.5505 \\
(0.0795)\end{array}$ & $\begin{array}{l}0.4443 \\
(0.0286)\end{array}$ & $\begin{array}{l}0.3726 \\
(0.0248)\end{array}$ & $\begin{array}{l}0.3012 \\
(0.0091)\end{array}$ \\
\hline$\lambda$ & $\begin{array}{l}9.7542 \\
(0.4159)\end{array}$ & $\begin{array}{l}1.2862 \\
(1.3960)\end{array}$ & $\begin{array}{l}1.5007 \\
(0.0991)\end{array}$ & $\begin{array}{l}1.0964 \\
(0.1634)\end{array}$ \\
\hline
\end{tabular}

Note: standard errors are in parenthesis. 
Table 6. Simulated 30-year Investment Returns under Myopic Loss Aversion

\begin{tabular}{lrrrr} 
& \multicolumn{2}{c}{ Group I } & \multicolumn{2}{c}{ Group II } \\
& \multicolumn{2}{c}{ Extreme Loss Averse } & \multicolumn{2}{c}{ Standard CPT } \\
\cline { 2 - 5 } Feedback & Mean & Std Dev & Mean & Std Dev \\
\hline Weekly & $84.61 \%$ & $5.07 \%$ & $537.26 \%$ & $313.77 \%$ \\
Monthly & $86.67 \%$ & $5.22 \%$ & $671.50 \%$ & $403.11 \%$ \\
Quarterly & $96.62 \%$ & $6.87 \%$ & $914.47 \%$ & $567.88 \%$ \\
Annually & $171.58 \%$ & $33.14 \%$ & $1180.33 \%$ & $757.21 \%$ \\
5-Yearly & $1180.69 \%$ & $757.51 \%$ & $1180.69 \%$ & $757.51 \%$
\end{tabular}


Figures

Figure 1. The Prospect Theory Value Function

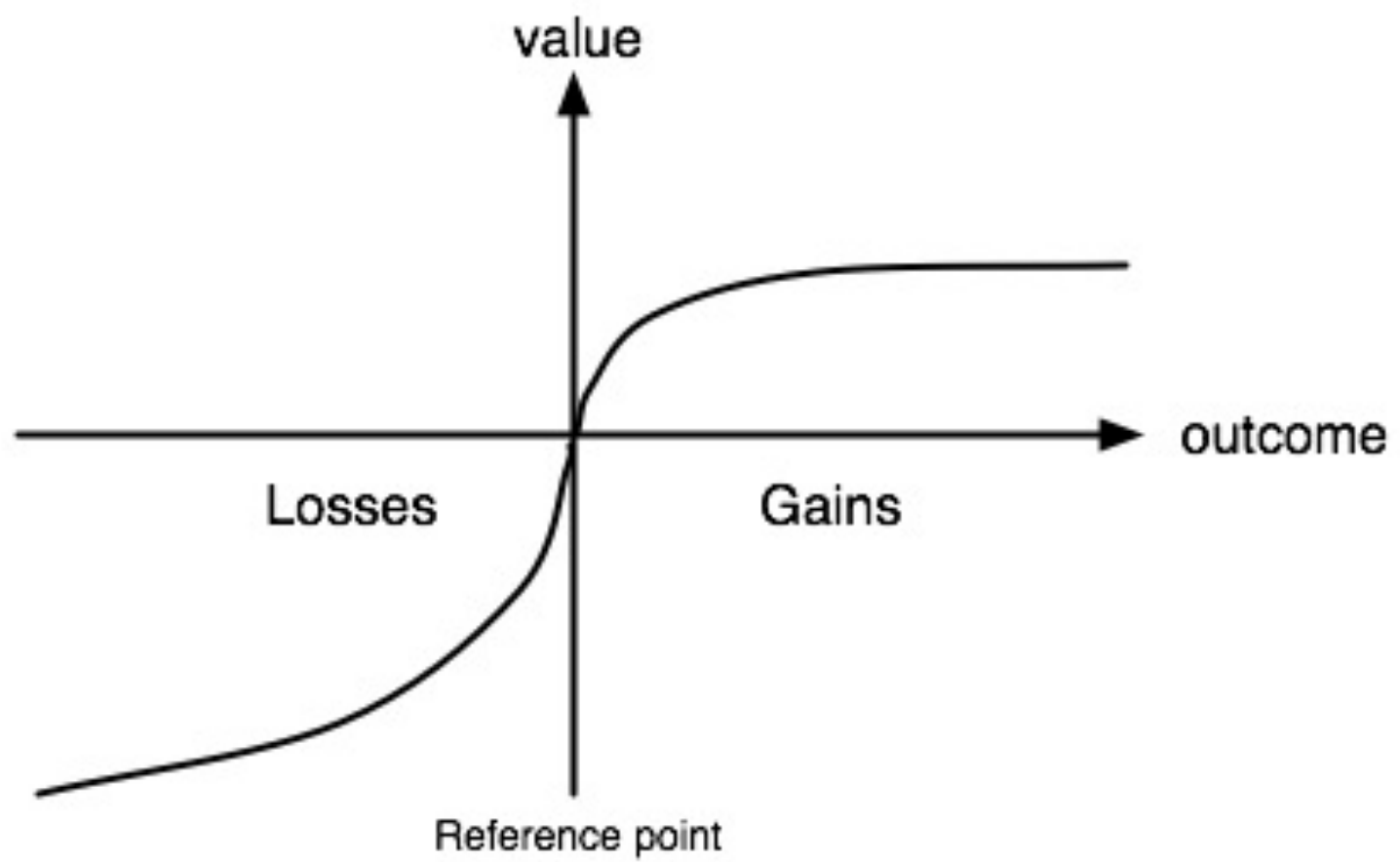


Figure 2. The Probability Weighting Function

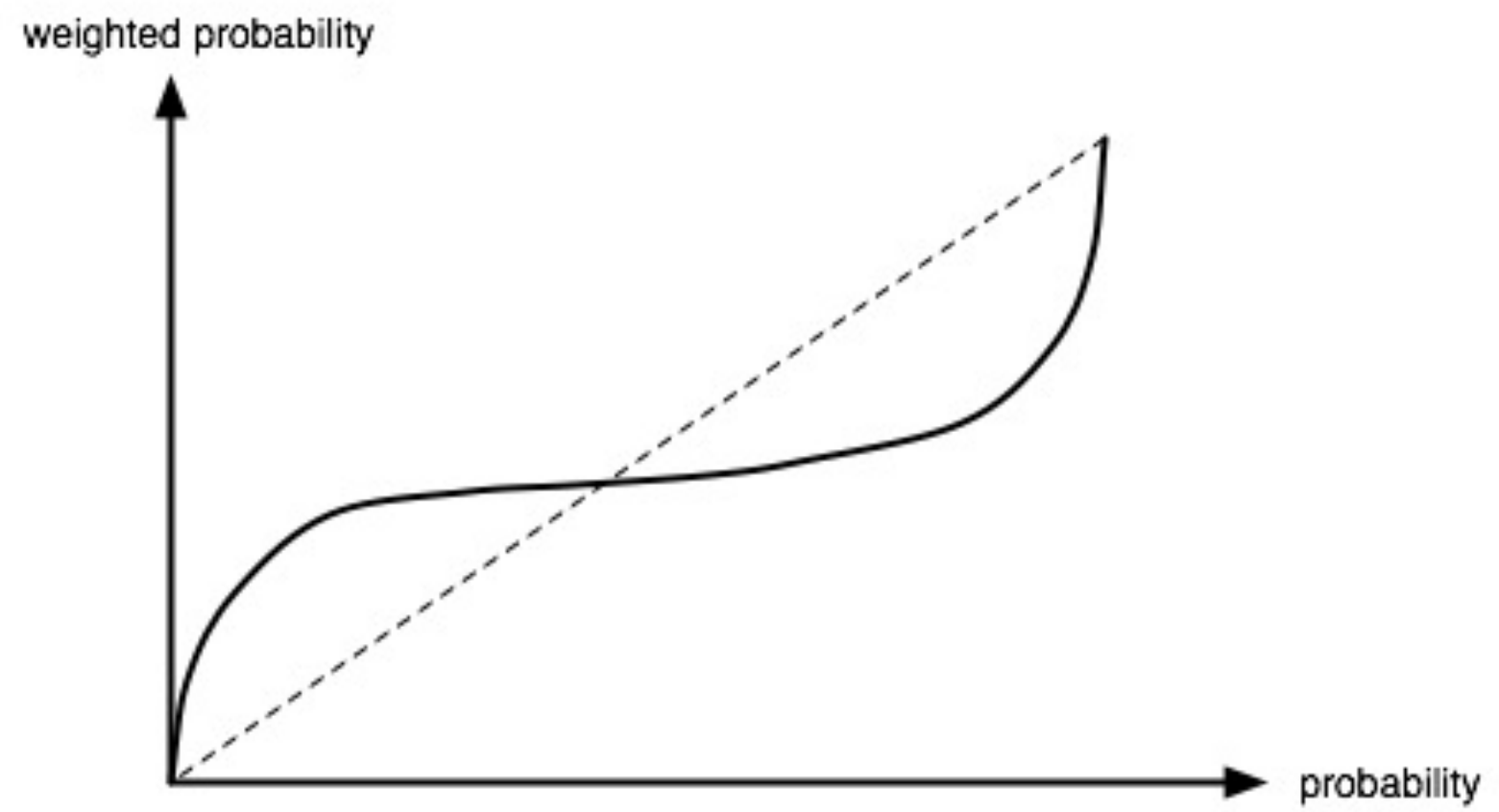


Figure 3. Posterior membership probabilities (2-Mixture)
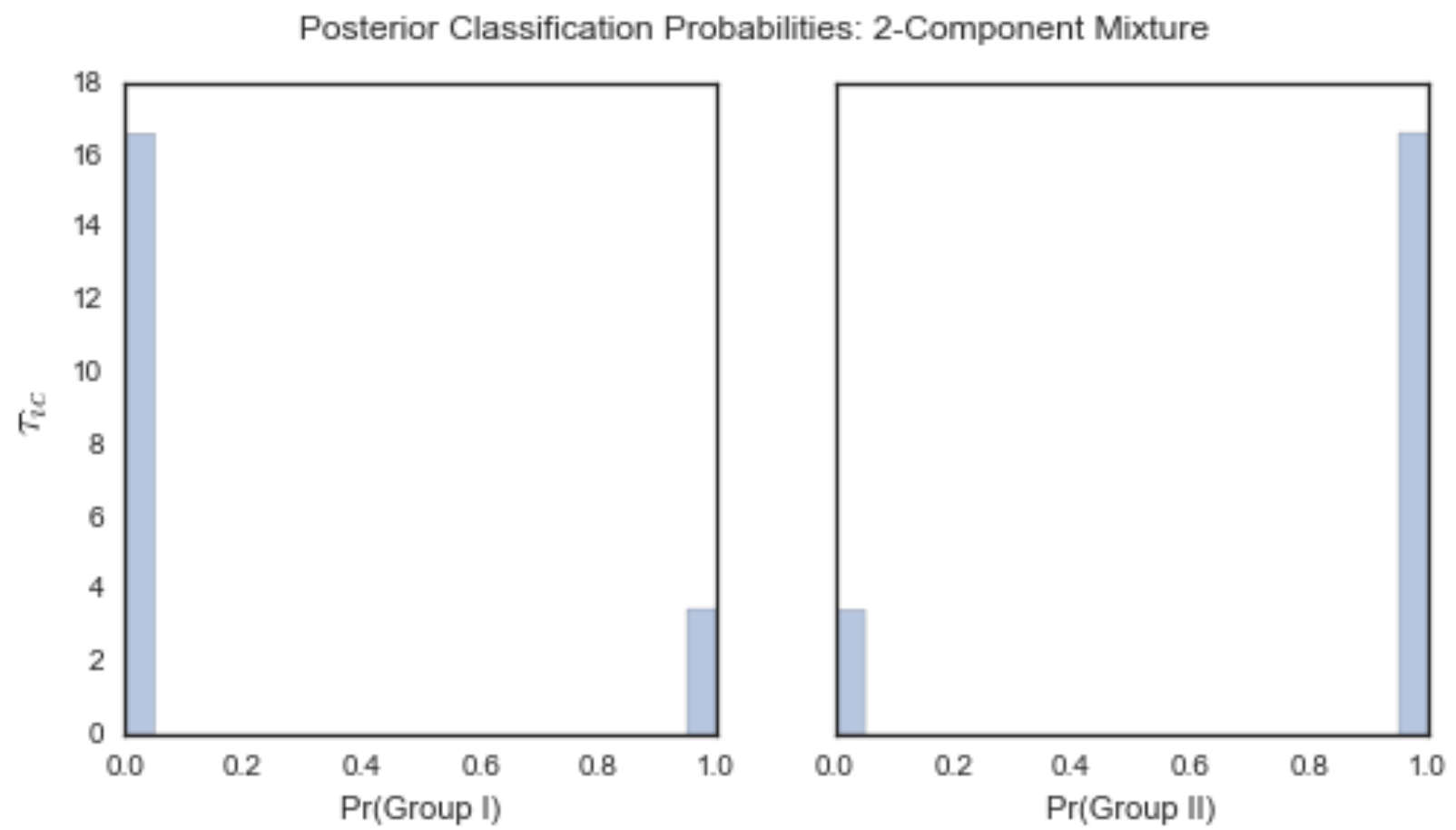
Figure 4. Posterior membership probabilities (4-Mixture)
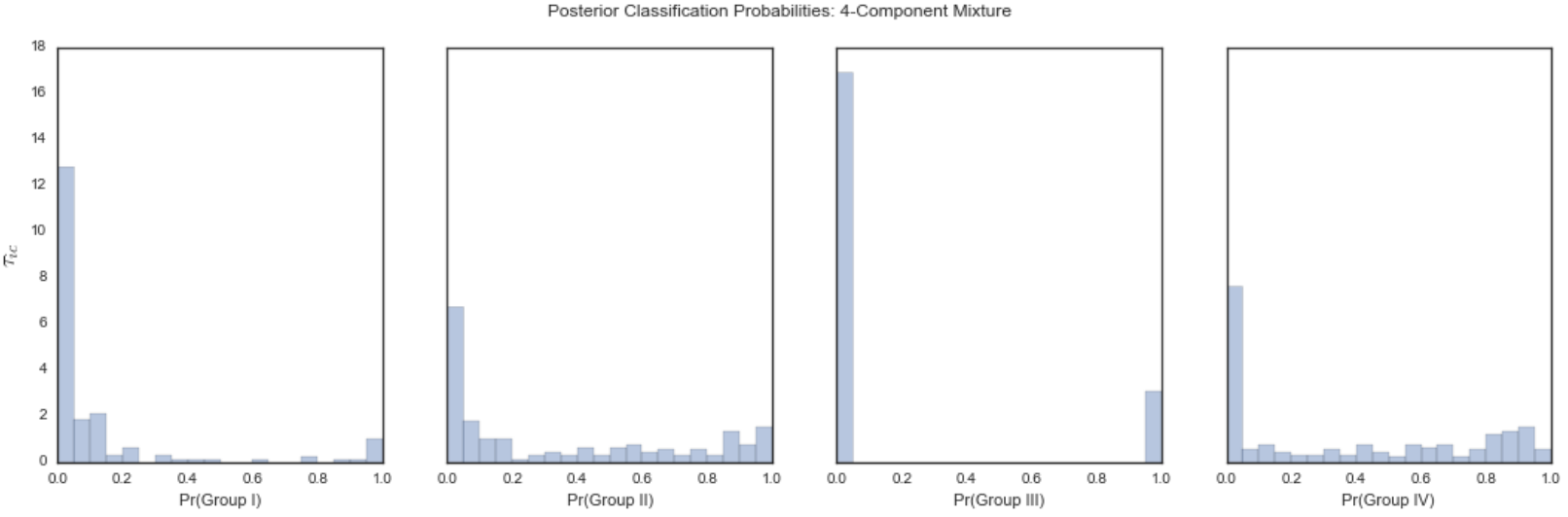
Figure 5. Marginal Kernel Densities by Model and by Parameter
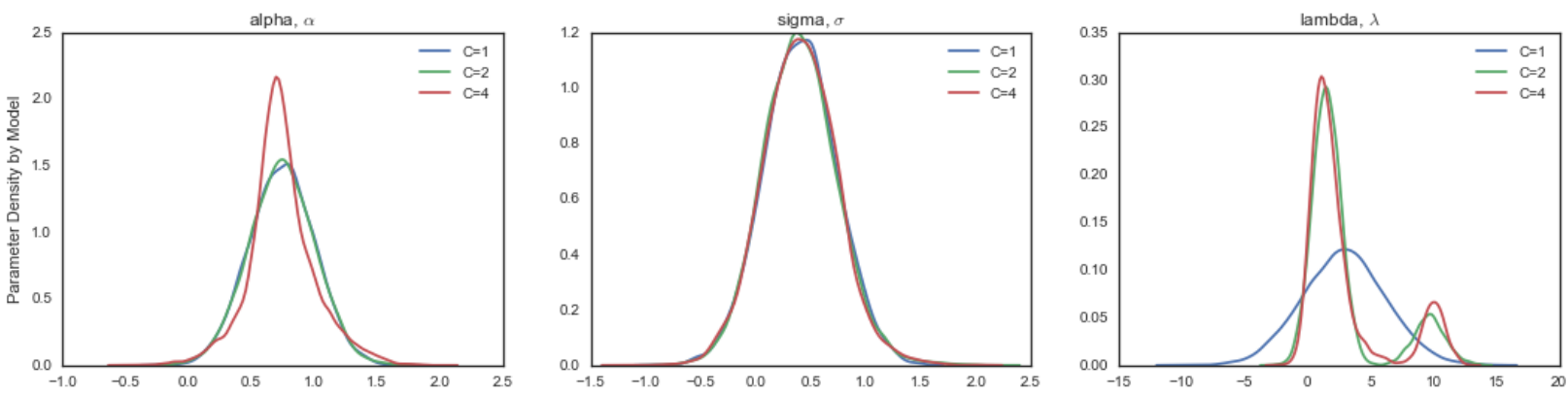
Figure 6. Histograms and Mixture Densities by Parameter (2-Mixture)

Estimation Results for the 2-Component Mixture
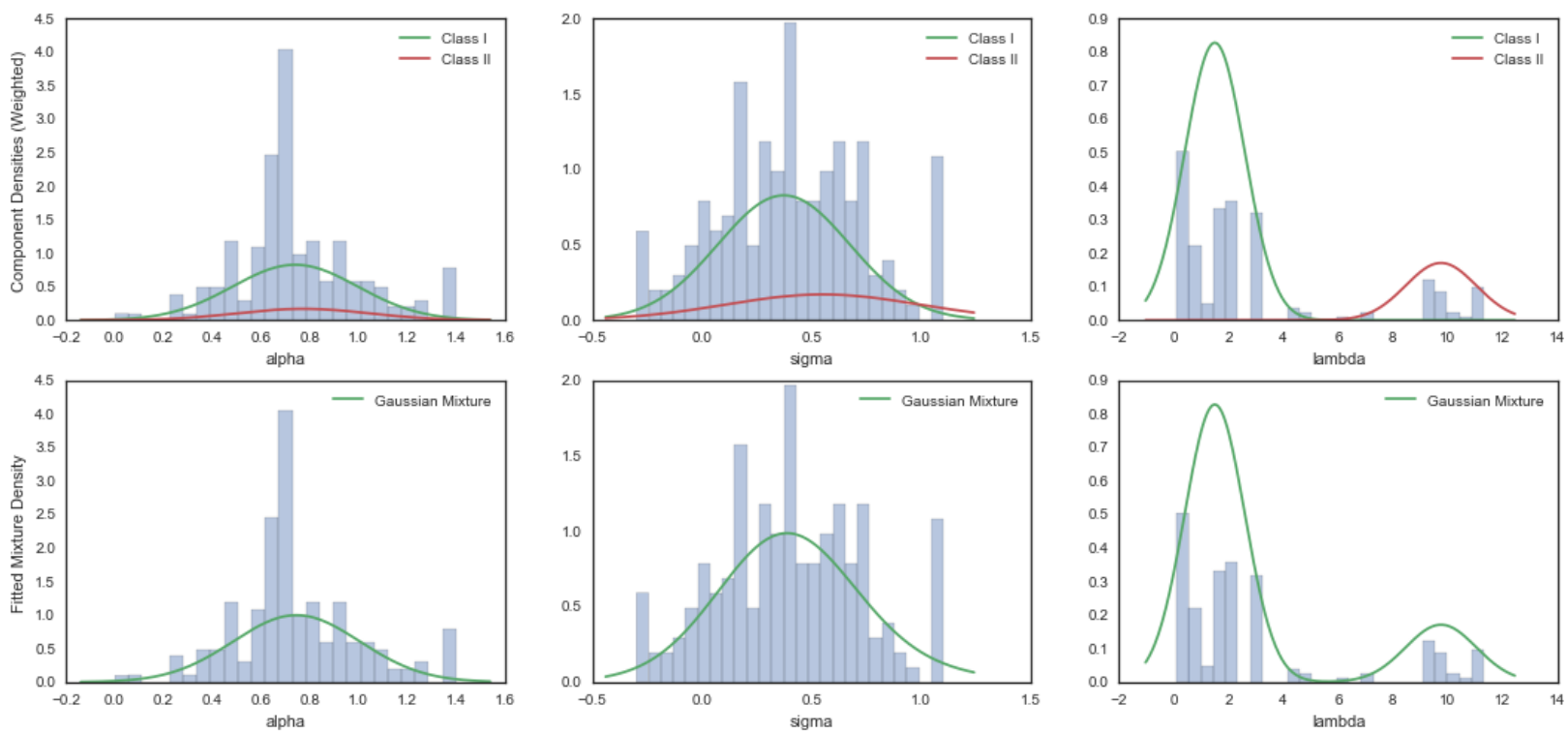
Figure 7. Effect of Linear Loss Aversion on Implied Equities Returns
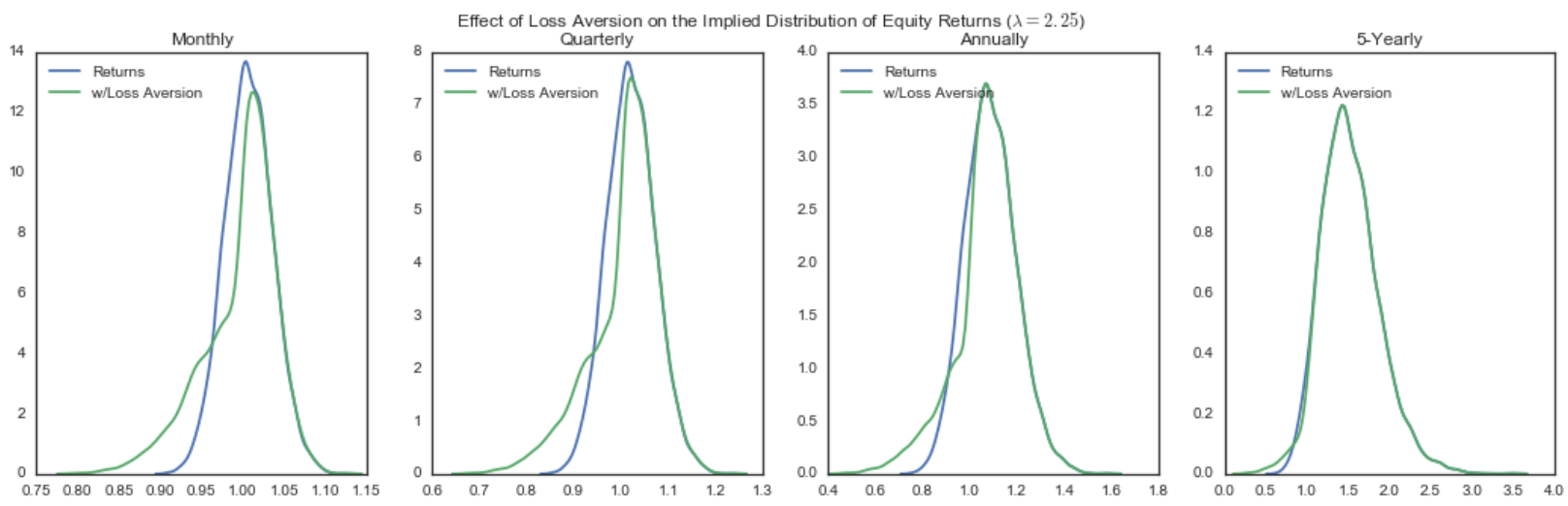
Figure 8. Investment Allocations by Feedback Frequency: 1-Mixture vs. 2-Mixture
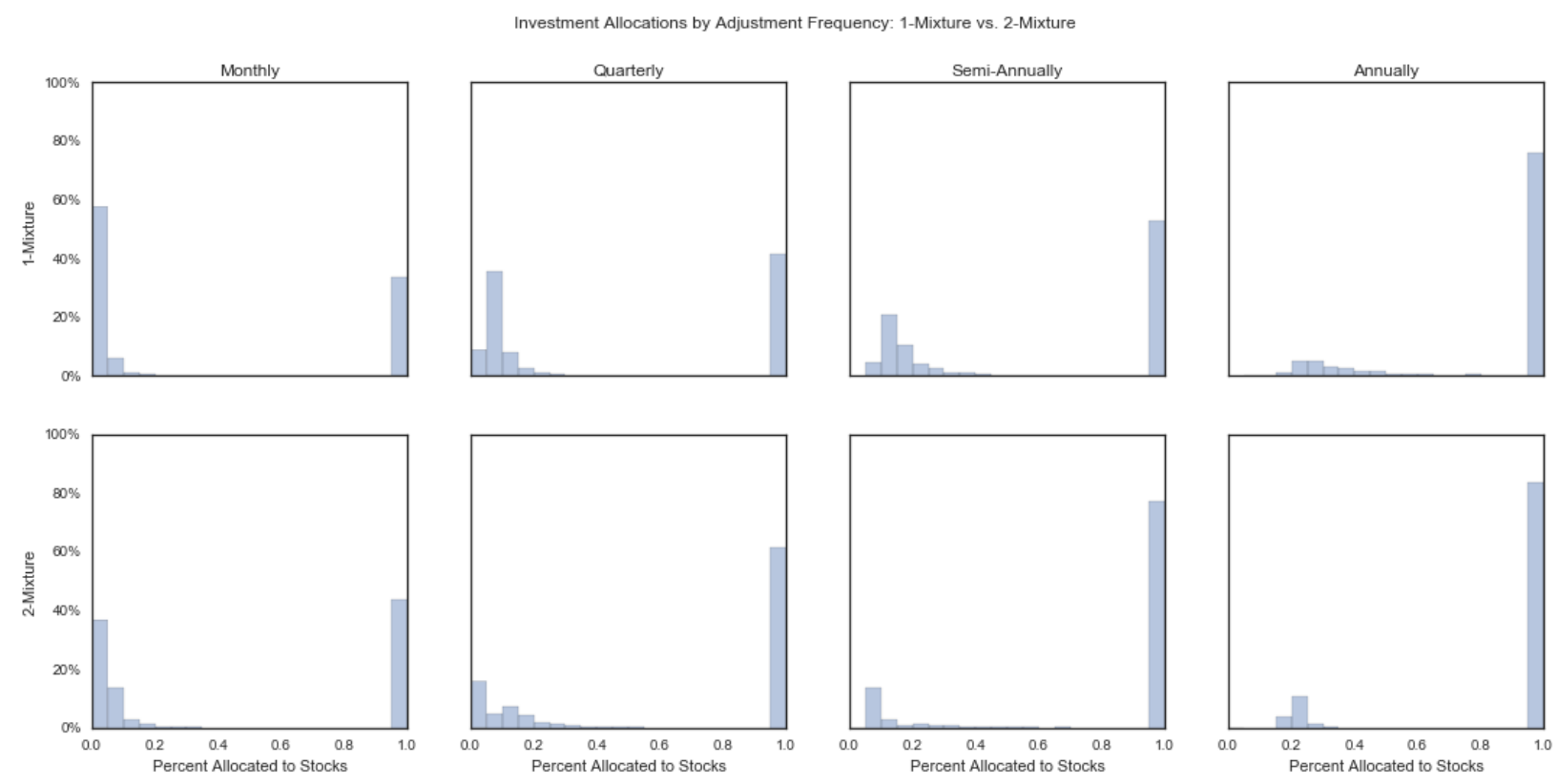